\title{
Dynamics of electric field screening in a bulk GaAs modulator
}

\author{
H. Heesel, S. Hunsche, H. Mikkelsen, T. Dekorsy, K. Leo, and H. Kurz \\ Institut für Halbleitertechnik II, Rheinisch-Westfälische Technische Hochschule Aachen, \\ Sommerfeldstrasse 24, W-5100 Aachen, Germany
}

\begin{abstract}
The transient development of electric-field distributions in a biased GaAs film after low density optical excitation is determined by measurements of Franz-Keldysh modulations with a time resolution of $100 \mathrm{fs}$. The experimental results are compared with theoretical calculations. The transient field is calculated with a drift-diffusion model. Our calculation of the dielectric function of $\mathrm{GaAs}$ includes the Coulomb coupling and the electric field. The resulting optical transmission changes are calculated with a transfer-matrix method. The theory predicts a modification of the FranzKeldysh modulation due to the nonuniform field, in quantitative agreement with the experimental observations.
\end{abstract}

Electromodulation spectroscopy ${ }^{1}$ is a well-established technique to obtain information about internal field strength, band gap, and composition of bulk semiconductors as well as of heterostructures. In particular, the screening of internal or external electric fields by optically injected carriers has been investigated by photoreflectance, ${ }^{2-4}$ electroreflectance, and electroabsorption measurements. ${ }^{5,6}$ In all these studies, the FranzKeldysh (FK) effect has been used to determine the electric field. Recently, there have been a number of publications which demonstrate that the nonuniformity of the electric fields has to be taken into account in order to get an accurate description of the experimental data. ${ }^{7,8}$

The investigation of the dynamics of electrical fields in semiconductors has recently gained much interest. A detailed knowledge of field screening dynamics is both important for a fundamental understanding of the transport dynamics of photoexcited carriers and for the application of semiconductor structures as optical modulators and ultrafast photonic switches. The dynamics of lateral $^{9-11}$ and vertical ${ }^{12}$ electrical fields due to photoexcited carriers has been studied in several experiments with subpicosecond time resolution. Recently, it was also demonstrated that the vertical transport of photoexcited carriers in surface space charge fields ${ }^{13}$ is accompanied by the emission of $\mathrm{THz}$ radiation. ${ }^{14}$

In this paper, we investigate the dynamics of the electric-field screening by photoexcited carriers in a bulk GaAs layer by measuring the differential transmission with a time resolution of $100 \mathrm{fs}$. Our basic intention is to quantitatively understand the temporal development of the electric field after above-band-gap excitation of free electrons and holes, which are accelerated and separated by the applied voltage. The internal field is screened through space-charge separation leading to a modulation of the dielectric function. The experimentally obtained differential transmission spectra show FK oscillations, which have a reduced amplitude for higher photon energies. A theoretical calculation of the carrier transport with a drift-diffusion model predicts that the electric-field screening exhibits a strong spatial modu- lation. Calculations of the optical spectra demonstrate that this nonuniformity is essential to explain the experimental data.

We perform pump-probe experiments using amplified $2-\mathrm{eV}$ pump pulses of 50 -fs duration and a white-light probe continuum in the range $1.3-1.9 \mathrm{eV}$ and measure the transmission spectra $T_{E}$ and $T_{0}$ of the probe beam with and without applied electric field at various time delays between pump and probe beam. The spectra are recorded with an optical multichannel analyzer (OMA III). The differential transmission spectra (DTS) are calculated as the normalized transmission change of the probe beam:

$$
\mathrm{DTS}=\left(T_{E}-T_{0}\right) / T_{0}
$$

The DTS are numerically corrected for the chirp of the probe continuum as previously described. ${ }^{15} \mathrm{By}$ this chirp correction the time resolution obtained is mainly determined by the duration of the pump pulse without any compression of the continuum probe pulses.

The experiments are performed on a $\mathrm{GaAs} / \mathrm{Al}_{x} \mathrm{Ga}_{1-x} \mathrm{As}$ double heterostructure grown by molecular-beam epitaxy on a (100)-oriented $n$-type GaAs substrate. The sample consists of a $0.2-\mu \mathrm{m}$-thick $i$ $\mathrm{Al}_{0.5} \mathrm{Ga}_{0.5} \mathrm{As}$ layer, a $0.22-\mu \mathrm{m}$-thick $i$-GaAs layer, a 0.5 $\mu \mathrm{m}$-thick $i$ - $\mathrm{Al}_{0.5} \mathrm{Ga}_{0.5} \mathrm{As}$ layer, and a $0.5-\mu \mathrm{m}$-thick $n$-type $\mathrm{Al}_{0.5} \mathrm{Ga}_{0.5} \mathrm{As}$ layer doped to $n=1 \times 10^{18} \mathrm{~cm}^{-3}$. A Schottky contact is formed by a $1-\mathrm{nm} \mathrm{Cr} / 5-\mathrm{nm}$ Au film on the frontside and a standard Ohmic contact to the substrate. The substrate is removed over an area of $2 \mathrm{~mm}^{2}$ by wet chemical etching to perform transmission measurements. The breakdown voltage of the diode is about $9 \mathrm{~V}$ corresponding to a maximal internal electric field of $90 \mathrm{kV} / \mathrm{cm}$. The actual sample structure is confirmed by measurements of static transmission and reflection spectra, which agree well with calculated ones. The Fabry-Pérot oscillations permit a precise determination of the actual layer thicknesses. This sample design prevents unwanted surface potentials as well as unintentional dopant diffusion out of the highly doped layer. We therefore assume a 
constant electric field in the active GaAs layer before the optical excitation. All pump-probe experiments are performed with an excitation density of $2 \times 10^{16} \mathrm{~cm}^{-3}$. The probe pulse intensity is kept low enough that the fieldinduced modulation of the spectra is not affected. Special care is taken to reduce the amplified spontaneous emission during the pump pulse, which can cause a photoinduced variation of the applied field even at negative delay times.

The solid lines in Fig. 1 show experimental DTS for different delay times between pump and probe pulse. The field-induced transmission change at zero time delay corresponds to the FK modulation caused by the initial field. With increasing delay time, the amplitude and the oscillation period of the modulation decrease. The modulation ratio for extrema at higher photon energy is strongly decreasing with increasing delay, so that the modulation is restricted to the region near the band edge for larger delay times. A residual field still exists for a delay time of $5 \mathrm{ps}$.

For a quantitative understanding of the experimental results, we need to calculate the theoretical DTS. The theory requires three steps: (i) simulation of the carrier transport after optical excitation to obtain the timedependent screening of the electric field inside the sample, (ii) calculation of the field and energy-dependent dielectric function, and (iii) calculation of the differential optical transmission.

For the calculation of the carriers transport, we use a one-dimensional drift-diffusion model for electrons and holes. ${ }^{13}$ The field distributions are obtained by solving the Poisson equation in a self-consistent manner. At the heterojunctions, the field is kept constant at the initial value because there is no current flow across the interfaces. The screening dynamics is entirely determined by

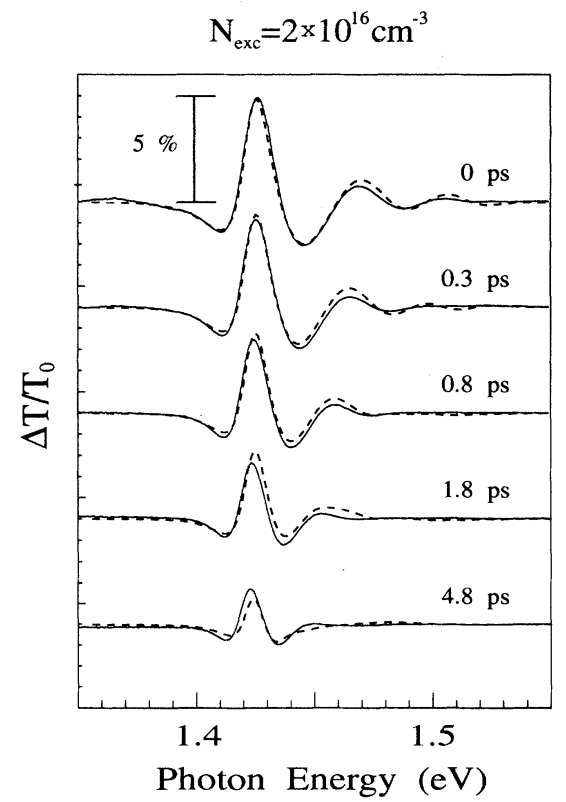

FIG. 1. Experimental and calculated DTS for different delay time. the carrier drift velocities, which are taken as the high field saturation values of $5 \times 10^{6} \mathrm{~cm} / \mathrm{s}$ for holes and $8 \times 10^{6}$ $\mathrm{cm} / \mathrm{s}$ for electrons, respectively. ${ }^{16}$ The effect of velocity overshoot ${ }^{12}$ is insignificant for optical excitation at $2 \mathrm{eV}$ and electrical field strengths $<50 \mathrm{kV} / \mathrm{cm} .^{17}$ Figure 2 shows the calculated electric fields across the active GaAs layer for different delay times and a carrier density of $1.5 \times 10^{16} \mathrm{~cm}^{-3}$ injected at $t=0$. For negative delay times, a homogeneous field of $34 \mathrm{kV} / \mathrm{cm}$ is present, which is determined by the sample thickness and applied voltage. For positive delay times, the internal field is screened by the space-charge field due to carrier separation. The asymmetry in field distribution over the layer arises from the difference of electron and hole saturation drift velocities. After 5 ps, a broad region of almost constant reduced field is established in the middle of the sample.

The electromodulation of the dielectric function of the GaAs layer is obtained by a detailed quantum-mechanical calculation of the Coulomb-enhanced absorption. The most simple approach would be using Airy functions and neglecting excitonic effects. ${ }^{18-20}$ We include excitonic effects in our calculation by closely following the theory of Blossey $^{21-23}$ and restrict ourselves to an outline of the procedure.

The absorption of a photon in a semiconductor results in the creation of an electron-hole pair, which is coupled by the Coulomb interaction. For a quantum-mechanical determination of the absorption strength, a correct description of these final states is required. It is obtained by solving numerically the time-independent Schrödinger equation describing the photoexcited electron-hole pair, including an external electric field and the Coulomb interaction between electron and hole:

$$
\left(-\frac{\hbar^{2}}{2 m_{e h}^{*}} \nabla^{2}-\frac{e^{2}}{4 \pi \epsilon_{0} \epsilon_{r}|\mathbf{r}|}+e F z\right) \phi_{n}(\mathbf{r})=E_{n} \phi_{n}(\mathbf{r}),
$$

where $m_{e h}^{*}$ is the reduced electron-hole effective mass, $\epsilon_{0} \epsilon_{r}$ is the static dielectric constant of the semiconductor (GaAs), $\mathbf{r}$ is the relative distance vector between the hole and the electron, and $z$ is the component of $\mathbf{r}$ along the $z$ axis, which is defined by the direction of the external electric field $F$. Finally, $E_{n}$ is the energy of the electronhole pair, and $\phi_{n}(\mathbf{r})$ is the combined electron-hole wave

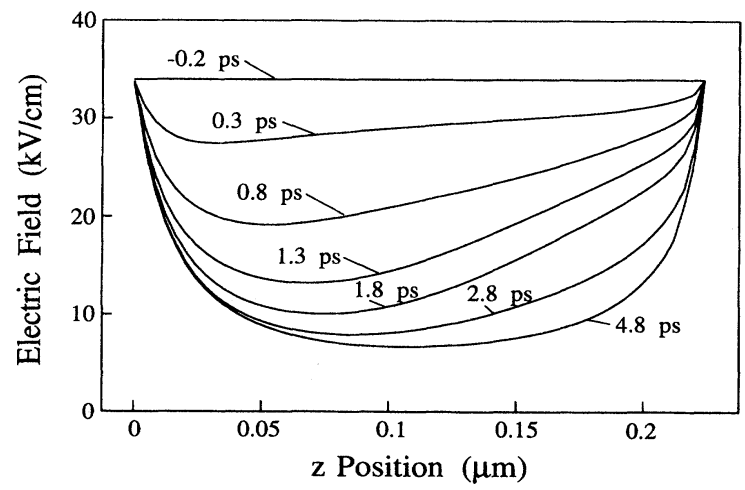

FIG. 2. Calculated $z$ distribution of electric fields in a GaAs film $(d=223 \mathrm{~nm})$ at different time delays. 
function. Equation (2) holds for direct transitions, i.e., for $\mathbf{k}_{e}+\mathbf{k}_{h} \cong 0$, where $\mathbf{k}_{e}$ and $\mathbf{k}_{h}$ are the wave vectors of electron and hole, respectively.

The Schrödinger equation is expressed in terms of dimensionless field-independent parameters and transformed with parabolic coordinates $\zeta=|\mathbf{r}|+z$ and $\eta=|\mathbf{r}|-z$, which allow separation into two independent equations. These are numerically integrated considering the correct asymptotic solutions to obtain the eigenvalues $E_{n}$ and eigenfunctions $\phi_{n}(\mathbf{r})$. From these, the densityof-states function $\phi^{2}(\omega)$ is calculated:

$\phi^{2}(\omega)=4 \pi^{2} a^{3} \sum_{n}\left|\phi_{n}(\mathbf{r}=\mathbf{0})\right|^{2} \delta\left[\left(E_{g}+E_{n}-\hbar \omega\right) / R\right]$,

with the exciton Rydberg constant $R$ and the exciton Bohr radius $a$. This finally gives the imaginary part of the dielectric function $\epsilon_{2}(\omega)$ :

$$
\epsilon_{2}(\omega)=\frac{4}{3} \epsilon_{0} \epsilon_{r}\left|\mu_{c v} / e a\right|^{2} \phi^{2}(\omega)
$$

where $\mu_{c v}$ is an interband dipole-matrix element and $\epsilon_{0} \epsilon_{r}$ is the static dielectric constant. To include collisional broadening ${ }^{3,8,24,25}$ the resulting spectra are convoluted with a Lorentz function with a full width half maximum of $7 \mathrm{meV}$.

In order to calculate the actual light transmission, we employ a transfer-matrix method ${ }^{26,27}$ for the electromagnetic wave equation. This allows to account for inhomogeneous field distributions resulting in different signal contributions from different depths of the active layer. The theoretical DTS for comparison with the experimental results are obtained according to Eq. (1). An inclusion of the light hole contribution ${ }^{2,8,20}$ would lead to a superposition of heavy-hole and light-hole oscillations for higher energies. We calculate the spectral deviation to be less than $2 \mathrm{meV}$ and thus neglect this effect in our model.

The dashed lines in Fig. 1 show theoretical calculations of the DTS using the complete theoretical method outlined above. The calculated and experimental spectra agree quantitatively. The temporal evolution of the amplitude as well as the oscillation period is well described. In the following, we discuss a comparison of the experimental results with calculations where nonuniform field distributions and excitonic effects have been neglected. This comparison shows that both effects are essential for a qualitative and quantitative agreement of experiment and theory.

The solution of the Schrödinger equation is given by Airy functions, if the Coulomb interaction between electrons and holes is neglected. Figure 3 shows a comparison of the calculated DTS with and without Coulomb interaction, using the same band gap of $E_{g}=1.428 \mathrm{eV}$. It is obvious that a quantitative agreement with the experimental data cannot be achieved by a simple Airy-function approach. The omission of the excitonic effects decreases the amplitude at the band edge by more than a factor of 2 and leads to a phase shift of the spectra above the band edge. The spectral position of the extrema as well as the decrease of oscillation amplitude for higher energies are

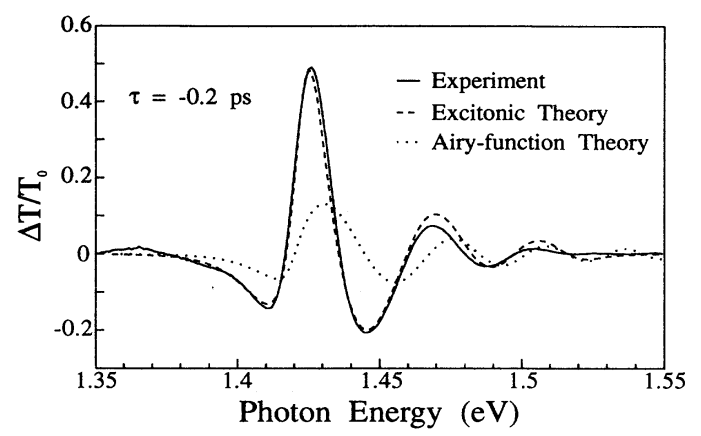

FIG. 3. Experimental and calculated DTS with and without Coulomb interaction.

only well reproduced by the full model.

Figure 4 shows a calculation assuming that the electric fields are homogeneous and switched between 0 and $15 \mathrm{kV} / \mathrm{cm}$. The experimentally observed signal reduction towards higher energies cannot be reproduced, which can be regarded as evidence that a nonuniform field is present in the GaAs layer. An alternative explanation for the damping of the FK oscillations at higher energies would be an energy-dependent damping constant. However, this explanation is immediately ruled out by the very good agreement between experiment and theory for zero time delay, when the field is homogeneous (see Fig. 3).

In conclusion, we have presented experimental and theoretical results for the time-dependent Franz-Keldysh modulation of the optical transmission of a thin GaAs film. The transient electric field has been calculated by a drift-diffusion model and is found to be nonuniform. The optical effect of field modulation is calculated from a Schrödinger equation including the Coulomb interaction potential. Theoretical spectra are calculated by a transfer-matrix method. We find a very good agreement between experiment and theory. To our knowledge, this is the first study which combines both a self-consistent calculation of the drift-diffusion transport and excitonic effects in the optical spectra to understand the time development of an inhomogeneous electric field in a semiconductor structure on a femtosecond time scale, which is of central importance for ultrafast photonic switching.

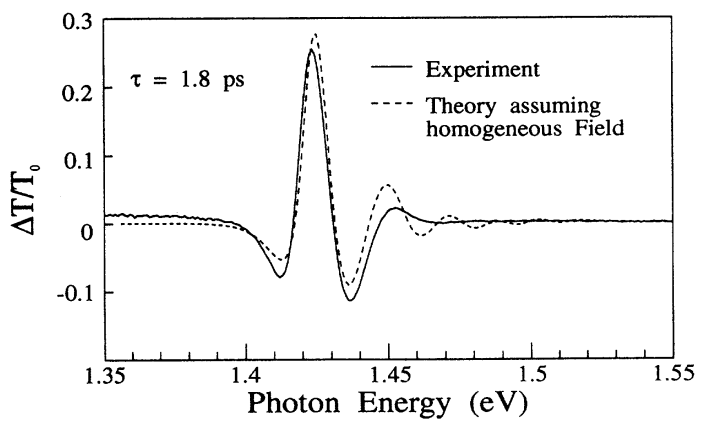

FIG. 4. Experimental DTS at $\tau=1.8 \mathrm{ps}$ compared with calculation assuming that the field is switched between $0 \mathrm{kV} / \mathrm{cm}$ and an average (homogeneous) field of $15 \mathrm{kV} / \mathrm{cm}$. 
The authors wish to thank K. Köhler for supplying the sample and D. Laschet for sample processing. Helpful discussions with J. T. Zettler and H. J. Bakker are gratefully acknowledged. This work was supported by the Volkswagen-Stiftung (Photonik-Schwerpunkt) and by the Alfried Krupp Stiftung. One of the authors (H.M.) has been supported through the Royal Norwegian Council for Scientific and Industrial Research (NTNF).
${ }^{1}$ M. Cardona, in Solid State Physics, Advances in Research and Applications, edited by F. Seitz, D. Turnbull, and H. Ehrenreich (Academic, New York, 1969).

2 C. Van Hoof, K. Deneffe, J. De Boeck, D.J. Arent, and G. Borghs, Appl. Phys. Lett. 54, 608 (1988).

${ }^{3}$ H. Shen and F.H. Pollak, Phys. Rev. B 42, 7097 (1990).

${ }^{4}$ X. Yin and F.H. Pollak, Appl. Phys. Lett. 56, 1278 (1990).

5 T.E. Eck, L.M. Walpita, W.S.C. Chang, and H.H. Wieder, Appl. Phys. Lett. 48, 451 (1986).

${ }^{6}$ A. Partovi and E.M. Garmire, J. Appl. Phys. 69, 6885 (1991).

${ }^{7}$ M. Sydor and J.R. Engholm, Appl. Phys. Lett. 56, 1769 (1990).

${ }^{8}$ R.A. Batchelor and A. Hamnett, J. Appl. Phys. 71, 2414 (1992).

${ }^{9}$ W.H. Knox, D.A.B. Miller, T.C. Damen, D.S. Chemla, and C.V. Shank, Appl. Phys. Lett. 48, 864 (1986).

${ }^{10}$ W.H. Knox, J.E. Henry, K.W. Gossen, K.D. Li, B. Tell, D.A.B. Miller, D.S. Chemla, A.C. Gossard, J. English, and S. Schmitt-Rink, IEEE J. Quantum Electron. 25, 2586 (1989).

${ }^{11}$ W. Sha, T.B. Norris, W.J. Schaff, and K.E. Meyer, Phys. Rev. Lett. 67, 2553 (1991).

12 C.V. Shank, R.L. Fork, and B.I. Greene, Appl. Phys. Lett. 38, 104 (1981).

${ }^{13}$ T. Dekorsy, T. Pfeifer, W. Kütt, and H. Kurz, Phys. Rev.
B 47, 3842 (1993).

${ }^{14}$ B.B. Hu, X.-C. Zhang, D.H. Auston, and P.R. Smith, Appl. Phys. Lett. 56, 506 (1990).

${ }^{15}$ T.F. Albrecht, K. Seibert, and H. Kurz, Opt. Commun. 84, 223 (1991).

${ }^{16}$ Semiconductors, edited by O. Madelung, LandoltBörnstein, New Series, Group III, Vol. 17, Pt. a (Springer, Berlin, 1982), p. 533.

${ }^{17}$ G.M. Wysin, D.L. Smith, and A. Redondo, Phys. Rev. B 38, 12514 (1988).

${ }^{18}$ D.E. Aspnes, Phys. Rev. 147, 554 (1966).

${ }^{19}$ D.E. Aspnes, Phys. Rev. 153, 973 (1967).

${ }^{20}$ F.C. Weinstein, J.D. Dow, and B.Y. Lao, Phys. Rev. B 4, 3502 (1971).

${ }^{21}$ D.F. Blossey, Phys. Rev. B 2, 3976 (1970).

${ }^{22}$ D.F. Blossey, Phys. Rev. B 3, 1382 (1971).

${ }^{23}$ R.K. Willardson and A.C. Beer, Semiconductors and Semimetals (Academic, New York, 1972), Vol. 9.

${ }^{24}$ T. Brudevoll, T.A. Fjeldly, J. Baek, and M.S. Shur, J. Appl. Phys 67, 7373 (1990).

25 J.A. Kash, J.C. Tsang, and J.M. Hvam, Phys. Rev. Lett. 54, 2151 (1985).

${ }^{26}$ J. Humelicek, Opt. Acta 30, 97 (1983).

${ }^{27}$ J.-Th. Zettler, H. Mikkelsen, K. Leo, H. Kurz, R. Carius, and A. Förster, Phys. Rev. B 46, 15955 (1992). 